\title{
EQUIVALENCE OF COCYCLES UNDER AN IRRATIONAL ROTATION
}

\author{
LARRY BAGGETT AND KATHY MERRILL
}

(Communicated by J. Marshall Ash)

\begin{abstract}
This paper describes a method for studying the equivalence relation among cocycles for an irrational rotation. A parameterized family of cocycles is presented, which meets the equivalence class of each piecewise absolutely continuous function whose derivative is $L^{2}$. The difficulties in describing the equivalence among the elements of this family is shown to reduce to the analogous problem for describing equivalence among step functions, thereby relating this paper to the earlier work of Veech, Petersen, Merrill, and others.
\end{abstract}

1. Introduction. In this paper we study the functional equations:

$$
v(x)=c+w(x)-w(x+\theta)
$$

and

$$
f(x)=\lambda g(x) / g(x+\theta)
$$

where $\theta$ is a fixed irrational number.

In (1), we are given a measurable real-valued function $v$ on $\mathbb{R} / \mathbb{Z}$ (which we identify with the half-open interval $[0,1)$ ), and we seek a real scalar $c$ and a measurable real-valued function $w$. In (2), we are given a measurable circle-valued function $f$ on $\mathbb{R} / \mathbb{Z}$, and we seek a complex scalar $\lambda$ and a measurable circle-valued function $g$.

If (1) has a solution, we say that $v$ is a trivial additive cocycle, and if (2) has a solution, then $f$ is a trivial multiplicative cocycle. Moore and Schmidt [4] have shown that $v$ is a trivial additive cocycle if and only if $e^{2 \pi i s v}$ is a trivial multiplicative cocycle for every real $s$. The resulting distinction between additive and multiplicative triviality has been studied in $[\mathbf{7}, \mathbf{3}$, and 6] among other places. Most of the results in this paper are for additive cocycles, although multiplicative techniques from the cited works will be used.

Two real-valued functions $v$ and $v^{\prime}$ are equivalent if $v-v^{\prime}$ is trivial. This equivalence relation and the parallel one for multiplicative cocycles are not smooth, meaning that there exists no Borel isomorphism between the set of equivalence classes and any standard Borel space. However, a need to understand this equivalence relation arises in many applications in harmonic analysis and ergodic theory (e.g., see [1, 2, and 8]).

Thus, as an alternative goal to parameterizing the equivalence classes completely, we seek a smoothly parameterized family $\mathbf{V}$ of real-valued measurable functions such that:

(a) every real-valued measurable function is equivalent to some element of $\mathbf{V}$,

Received by the editors February 18, 1988.

1980 Mathematics Subject Classification (1985 Revision). Primary 11K38, 22D30, 28 D05.

This research was supported in part by NSF grants DMS8600753 and DMS8401052. 
and

(b) the equivalence relation on $\mathbf{V}$ can be described in terms of the parameters of $\mathbf{V}$.

The only partial result of this kind that we know of is in [1]. There, the multiplicative family $\mathbf{V}$ consists of all translates of exponential functions $e_{s}$, where $e_{s}(x)=e^{2 \pi i s x}$. This family satisfies property (b) but not (a). In this paper we propose a much broader additive family.

Throughout the rest of this paper we will assume that $\theta$ is a badly approximable irrational, i.e., that there exists a positive constant $\delta$ such that

$$
n\left|1-e^{2 \pi i n \theta}\right| \geq \delta
$$

for all positive integers $n$. This is equivalent to assuming that $\theta$ has bounded partial quotients in its continued fraction expansion, a condition that has been seen $[\mathbf{7}, \mathbf{1}]$ to simplify the equivalence relation we are studying.

2. Let $\mathbf{V}$ be the family of piecewise linear, real-valued functions on $\mathbf{R} / \mathbf{Z}$. We parameterize $\mathbf{V}$ as follows: For each partition $t_{1}<\cdots<t_{N}$ of $[0,1)$, and for each $N$-tuple $\left(l_{1}, r_{1}\right),\left(l_{2}, r_{2}\right), \cdots,\left(l_{N}, r_{N}\right)$ of elements of $\mathbf{R}^{2}$, define $v^{\left\{t_{i}\right\},\left\{\left(l_{i}, r_{i}\right)\right\}}$ to be linear on $\left(t_{i}, t_{i+1}\right), 1<i<N$, and on $\left(t_{N}, t_{1}\right)$, with left and right limits at $t_{i}$ equal to $l_{i}$ and $r_{i}$ respectively. We will show that every piecewise absolutely continuous function with $L^{2}$ derivative is equivalent to some element of $\mathbf{V}$, and we will discuss the equivalence relation among the elements of $\mathbf{V}$.

LEMMA 2.1. If $v$ is absolutely continuous with $v^{\prime}$ in $L^{2}$, then $v$ is a trivial additive cocycle.

PROOF. Let $\left\{c_{n}\right\}$ be the Fourier coefficients for $v$. By integration by parts, $\left\{n c_{n}\right\}$ is in $l^{2}$. Let $a_{n}=c_{n} /\left(1-e^{2 \pi i n \theta}\right)$ for $n \neq 0$, and $a_{0}=0$. Then since $\theta$ is badly approximable, $\left\{a_{n}\right\}$ is also in $l^{2}$. Let $w(x)=\sum a_{n} e^{2 \pi i n x}$. Then $w(x)-w(x+\theta)+c_{0}$ is an $L^{2}$ function with Fourier coefficients $\left\{c_{n}\right\}$, and thus equals $v$.

DEFINITION. A function $v:[0,1) \rightarrow \mathbf{R}$ is piecewise absolutely continuous with $L^{2}$ derivative if there exists a partition $t_{1}<\cdots<t_{N}$ of $[0,1)$ such that $v$ is absolutely continuous on each interval $\left(t_{i}, t_{i+1}\right)$, and on $\left(t_{N}, t_{1}\right)$, and if its a.e. derivative $v^{\prime}$ is in $L^{2}$.

For such a function $v$, it follows that left and right limits $v\left(x^{-}\right)$and $v\left(x^{+}\right)$exist at every point in $[0,1)$, whence the discontinuities of $v$ are jump discontinuities.

THEOREM 2.2. Every piecewise absolutely continuous function $v$ with $L^{2}$ derivative is equivalent to some element of $\mathrm{V}$.

ProOF. Let $t_{1}<\cdots<t_{N}$ be a partition of $[0,1)$ which contains all the discontinuities of $v$, and let $\left(l_{i}, r_{i}\right)=\left(v\left(t_{i}^{-}\right), v\left(t_{i}^{+}\right)\right)$. Then $v-v^{\left\{t_{i}\right\},\left\{\left(l_{i}, r_{i}\right)\right\}}$ is an absolutely continuous function with $L^{2}$ derivative, and thus trivial.

Now we explore the equivalence relation among piecewise linear functions. The first lemma shows that every piecewise linear function is equivalent to one which has nonzero slope on at most one of its subintervals:

LEMMA 2.3. Let $v=v^{\left\{t_{i}\right\},\left\{\left(l_{i}, r_{i}\right)\right\}}$ be a piecewise linear function. Then $v$ is equivalent to any piecewise linear function of the form $v^{\left\{t_{i}\right\},\left\{\left(l_{i}^{\prime}, r_{i}^{\prime}\right)\right\}}$ where the jumps $r_{i}-l_{i}$ and $r_{i}^{\prime}-l_{i}^{\prime}$ are equal for $1 \leq i \leq N$, and where $l_{i+1}^{\prime}=r_{i}^{\prime}$ for $1 \leq i<N$. 
PROOF. $v^{\left\{t_{i}\right\},\left\{\left(l_{i}, r_{i}\right)\right\}}-v^{\left\{t_{i}\right\},\left\{\left(l_{i}^{\prime}, r_{i}^{\prime}\right)\right\}}$ is an absolutely continuous function with $L^{2}$ derivative, and thus trivial.

Note that the location and size of the jump discontinuities of $v$ are invariant under the equivalences in 2.2 and 2.3. The following theorem gives a necessary condition for triviality in terms of these invariants.

THEOREM 2.4. A piecewise linear function is trivial only if the sum of its jumps, $\sum\left(r_{i}-l_{i}\right)=0$.

PROOF. Let $v=v^{\left\{t_{i}\right\},\left\{\left(l_{i}, r_{i}\right)\right\}}$ be such that the sum of its jumps is nonzero, and suppose $v$ is trivial. By 2.3, we can assume that $v$ has slope 0 on $\left(t_{i}, t_{i+1}\right)$ for $0<i<N$ and slope $m \neq 0$ on $\left(t_{N}, t_{1}\right)$. For any integer $n$, define

$$
v^{(n)}(x)=v(x)+v(x+\theta)+\cdots+v(x+(n-1) \theta) .
$$

Since $v$ is trivial, it follows (see [7 or 3]). that for any sequence $\left\{n_{k}\right\}$ with $n_{k} \theta \rightarrow 0$ mod 1 , there exists a complex number $\mu$ of modulus 1 , and a subsequence $\left\{n_{k_{j}}\right\}$ for which $\lim f_{j}(x)=\mu$ for almost all $x$, where $f_{j}=e^{2 \pi i v_{j}}$, and $v_{j}=v^{\left(n_{k_{j}}\right)}$. We will select a $j$ with properties that lead to a contradiction.

Let $\varepsilon=\left(1-\left(t_{N}-t_{1}\right)\right)|m| / 20 N$, and let $E_{j}$ be the set of $x$ for which $\left|\mu-f_{j}(x)\right|<\varepsilon$. Then $\lim \nu\left(E_{j}\right)=1$, where $\nu$ is Lebesgue measure. Choose first a $j$ such that $\nu\left(E_{j}\right)>4 / 5$. Let $\left\{I_{j, r}\right\}$ be the set of intervals between discontinuities of $v_{j}$. If $I_{j, r}$ is one of these intervals, then $v_{j}$ is linear on $I_{j, r}$, with slope an integer $p_{j, r}$ times $m$. The interval $v_{j}\left(I_{j, r}\right)$ is of length $\nu\left(v_{j}\left(I_{j, r}\right)\right)=p_{j, r}|m| \nu\left(I_{j, r}\right)$. We will reach a contradiction by showing that for many intervals $I_{j, r}, v_{j}\left(I_{j, r}\right)$ is too large for $f_{j}$ to stay within $\varepsilon$ of $\mu$.

First we estimate $p_{j, r}$. It is exactly the number of integers $0<h<n_{k_{j}}$ such that $x+h \theta \in\left[t_{N}, t_{1}\right)$, where $x$ is any point in $I_{j, r}$. The Ergodic Theorem then implies that for almost all $x$,

$$
\lim _{j} \frac{p_{j, r}}{n_{k_{j}}}=\left|t_{1}-t_{N}\right|=1-\left(t_{N}-t_{1}\right) .
$$

We may choose our $j$ so that, in addition to the first requirement, $p_{j, r}>$ $n_{k_{j}}\left(1-\left(t_{N}-t_{1}\right)\right) / 2$ for all $x$ in a set $F_{j}$ for which $\nu\left(F_{j}\right)>.9$. Now we estimate the length of $I_{j, r}$. The average length of the intervals $\left\{I_{j, r}\right\}$ is at least $1 / N n_{k_{j}}$, and the sum of the lengths of those intervals, whose length is at least half this average, is at least $1 / 2$. Let $G_{j}$ be the set of all $x \in F_{j}$ such that the interval $I_{j, r}$ to which $x$ belongs has length at least half the average length. Then $\nu\left(G_{j}\right) \geq .4$, and $\nu\left(E_{j} \cap G_{j}\right) \geq .2$.

Now, if $x \in E_{j} \cap G_{j}$, and if $I_{j, r}$ is the interval in $\left\{I_{j, r}\right\}$ to which $x$ belongs, then

$$
\nu\left(v_{j}\left(I_{j, r}\right)\right) \geq n_{k_{j}}\left(1-\left(t_{N}-t_{1}\right)\right)|m| / 4 N n_{k_{j}}=\left(1-\left(t_{N}-t_{1}\right)\right)|m| / 4 N=5 \varepsilon \text {. }
$$

It follows that $\nu\left(I_{j, r} \cap E j\right) \geq .6 \nu\left(I_{j, r}\right)$, whence

$$
\nu\left(E_{j}\right) \geq \nu\left(E_{j} \cap G_{j}\right) \geq .6 \nu\left(G_{j}\right) \geq .24,
$$

and this gives the desired contradiction.

Because of Theorem 2.2 (and the remark following 2.3), Theorem 2.4 applies as well to piecewise absolutely continuous functions with $L^{2}$ derivatives. Note also that if the sum of the jumps of such a function is 0 , Lemma 2.3 will produce an equivalent step function. Thus we have the following alternate version of Theorem 2.4: 
THEOREM 2.5. A piecewise absolutely continuous function with $L^{2}$ derivative which is a trivial additive cocycle must be equivalent to a step function.

COROLLARY. A piecewise absolutely continuous function with $L^{2}$ derivative, which has exactly one point of discontinuity on $\mathbb{R} / \mathbb{Z}$, is a nontrivial additive cocycle.

Thus, for example, a nonconstant increasing Lipschitz continuous function on $[0,1)$ cannot be trivial.

Examples in $[3,5$, and 7], show that the conditions given in 2.4 and 2.5 are not sufficient for triviality. Thus we have not described completely the equivalence relation on $\mathbf{V}$; instead, we have reduced the problem of determining equivalence in $\mathbf{V}$ to the admittedly still difficult problem of determining equivalence among step functions. However, we can make use of a partial result known for step functions to give a necessary and sufficient condition for triviality in the following restricted family $\mathbf{V}^{\prime}$ :

DEFINITION. The family $\mathbf{V}^{\prime} \subset \mathbf{V}$ consists of all functions $v^{\left\{t_{i}\right\},\left\{\left(l_{i}, r_{i}\right)\right\}}$ for which no proper subsum of the jumps $r_{i}-l_{i}$ is 0 .

We see as before that any piecewise absolutely continuous function with $L^{2}$ derivative, which satisfies the same subsum property, will be equivalent to a function in $\mathbf{V}^{\prime}$.

THEOREM 2.6. An element $v^{\left\{t_{i}\right\},\left\{\left(l_{i}, r_{i}\right)\right\}} \in \mathbf{V}^{\prime}$ is trivial if and only if

(a) $\sum\left(r_{i}-l_{i}\right)=0$, and

(b) each $t_{i+1}-t_{i}$ is of the form $p_{i}+q_{i} \theta, 1 \leq i<N-1$.

ProOF. Suppose $v$ satisfies (a) and (b). Then, as in $2.5, v$ is equivalent to a step function satisfying (b), which is trivial [ 7 or 3 ].

Conversely, suppose $v \in \mathbf{V}^{\prime}$ is trivial. Then by 2.4, it satisfies (a), and by 2.5 it is equivalent to a step function. Now we apply Theorem 3.1 of [3] to show that $e^{2 \pi i v}$ can be trivial only if (b) holds as well.

\section{REFERENCES}

1. L. Baggett and K. Merrill, Representations of the Mautner group and cocycles of an irrational rotation, Michigan Math. J. 33 (1986), 221-229.

2. H. Helson, Analyticity on compact abelian groups, Algebras In Analysis, Academic Press, (1975), pp. 1-62.

3. K. Merrill, Cohomology of step functions under irrational rotations, Israel J Math. 52 (1985), pp. 320-340.

4. C. C. Moore and K. Schmidt, Coboundaries and homomorphisms for nonsingular actions and a problem of H. Helson, Proc. London Math. Soc. 40 (1980), 443-475.

5. K. Petersen, On a series of cosecants related to a problem in ergodic theory, Compositio Math. 26 (1973), 313-317.

6. M. Stewart, Irregularities of uniform distribution, Acta Math. Sci. Hungar. 37 (1981), 185-221.

7. W. A. Veech, Strict ergodicity in zero-dimensional dynamical systems and the Kronecker-Weyl theorem, mod 2, Trans. Amer. Math. Soc. 140 (1969), 1-33.

8. __ Finite group extensions of irrational rotations, Israel J. Math. 21 (1975), 240-259.

Department of MAThematics, CAMpus BoX 426, University of Colorado, BOUldER, COLORADO 80309

Department of Mathematics, The Colorado College, Colorado Springs, COLORADO 80903 